\title{
Multipactoring zone map of an rf input coupler and its application to high beam current storage rings
}

\author{
Tetsuo Abe, ${ }^{*}$ Tatsuya Kageyama, Kazunori Akai, Kiyokazu Ebihara, Hiroshi Sakai, and Yasunao Takeuchi \\ Accelerator Laboratory, High Energy Accelerator Research Organization (KEK), Tsukuba, Ibaraki 305-0801, Japan
} (Received 16 November 2005; published 20 June 2006)

\begin{abstract}
Multipactoring phenomena in a radio frequency (rf) input coupler, which could cause serious problems during accelerator operation, are described based on electrodynamics with the superposition state of the input and reflected waves in the coupler. Since the reflection coefficient varies widely with the beam loading in the case of high beam current storage rings, multipactoring zones should be represented in the two-dimensional space of the input rf power and the reflection coefficient. Based on this map, we have performed a simulation study for normal-conducting rf accelerating cavities and developed a method to operate an rf cavity with multipactoring problems so as to minimize the influence of multipactoring.
\end{abstract}

DOI: 10.1103/PhysRevSTAB.9.062002

PACS numbers: 29.20.Dh, 29.20.Lq

\section{INTRODUCTION}

The ARES [1-3], standing for accelerator resonantly coupled with energy storage, is a normal-conducting radio frequency (rf) accelerating cavity system for the KEK Bfactory (KEKB) [3-12]. This system makes it possible for KEKB to store high beam currents of electrons and positrons stably. This stability is obtained by having an additional energy-storage cavity with a large cylindrical shape, which is electromagnetically coupled, via a small coupling cavity, to the accelerating cavity with a structure for damping higher order modes; therefore, the ARES is a unique three-cavity system.

An input coupler is a device used to feed the rf power from a klystron into a cavity, which is one of the most important components for high-power rf systems. In the ARES, coaxial-line couplers are used with a loop for coupling to the magnetic field of the $\mathrm{TE}_{013}$ mode in the energy-storage cavity. A schematic drawing is shown in Fig. 1. The ARES input couplers have been tested in a coupler test stand up to or over $800 \mathrm{~kW}$ time-averaged power of the continuous wave $[13,14]$.

The tested couplers have been used in KEKB operations, where 20 ARES cavities are operating to store a $3.5-\mathrm{GeV}$ positron beam in the low-energy ring, and 12 ARES cavities together with eight superconducting cavities (SCCs) to store an $8.0-\mathrm{GeV}$ electron beam in the high-energy ring (HER). In 2003, the vacuums in two of the ARES cavities for the HER deteriorated significantly compared with those in the other cavities. The vacuum pressures rose up to several times higher values at certain input-power levels with and without a beam. From this dependence of the vacuum pressures on the input rf power and discharge phenomena observed with a TV camera through the inside of the cavity, it turned out that the vacuum-pressure rises resulted from resonant electron multiplication in the coaxial line of the input couplers. This phenomenon arises from

*Electronic address: tetsuo.abe@kek.jp secondary electron emissions after impact of primary electrons and repetition of this process driven by the rf field, known as multipactoring (or multipacting). Since there was no conditioning effect, we replaced the couplers by new ones, which had passed high-power tests without multipactoring symptoms. In addition, we scraped and wiped the inner surfaces of the coupler ports to remove the possibility of multipactoring sources in the cavities. However, one of the two cavities still exhibited the multipactoring problem.

Usually, multipactoring in a coaxial line can be suppressed by using the DC electric bias method $[15,16]$. However, the ARES coupler has a coupling loop at the end of the coaxial line, so that no DC bias can be applied.

In order to find a solution, we have performed a simulation study of multipactoring, where the strength of multipactoring is calculated as a function of not only the input rf power but also the reflection coefficient $(\Gamma)$ because $\Gamma$

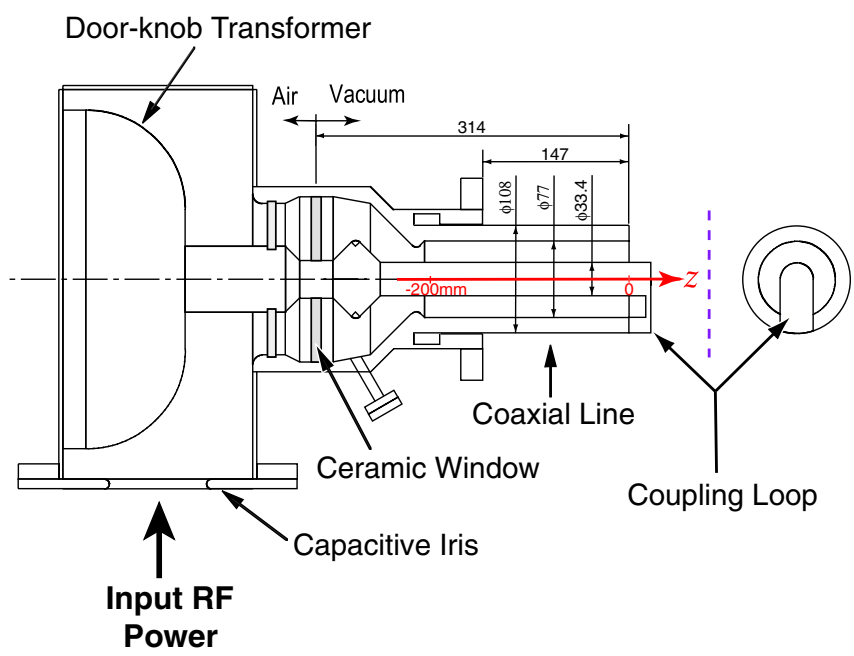

FIG. 1. (Color) Schematic drawing of the ARES input coupler. The $z$ axis used in the multipactoring simulation is shown as a horizontal arrow. The vertical dashed line indicates the position of the effective reflection plane explained in the text. 
varies widely with the beam loading, including both the overcoupling $(\Gamma>0)$ and undercoupling $(\Gamma<0)$ regions, in the case of high beam current storage rings, such as KEKB. It should be noted that the phase as well as the amplitude of the reflected wave have to be taken into account in the multipactoring simulation. It should be also noted that the reflected power together with the input rf power is used in [17] to represent the multipactoring zones, while we adopt the reflection coefficient instead of the reflected power due to the reason mentioned above. Furthermore, we have developed a method to operate the above-mentioned $\mathrm{rf}$ cavity with the multipactoring problem, based on a multipactoring zone map, for the accelerator performance not to be degraded, by minimizing the influence of the multipactoring.

In the next section, the method of the multipactoring simulation and the zone map are explained, followed by an application of the map to an operational strategy against multipactoring.

\section{MULTIPACTORING ZONE MAP}

\section{A. Multipactoring simulation}

In this paper, cylindrical coordinates $(r, \theta, z)$ are used with the $z$ axis taken along the axis of the coaxial line, where the origin is located at the end, as shown in Fig. 1. The region of the coupler coaxial line for multipactoring is defined as $-200 \mathrm{~mm}<z<-50 \mathrm{~mm}$. The region of $z>$ $-50 \mathrm{~mm}$ is excluded due to the perturbation effect of the coupling loop on the TEM mode, which was determined using a three-dimensional electromagnetic simulation. Examples of the simulation results are shown in Fig. 2, illustrating the perturbation effect.

(a)

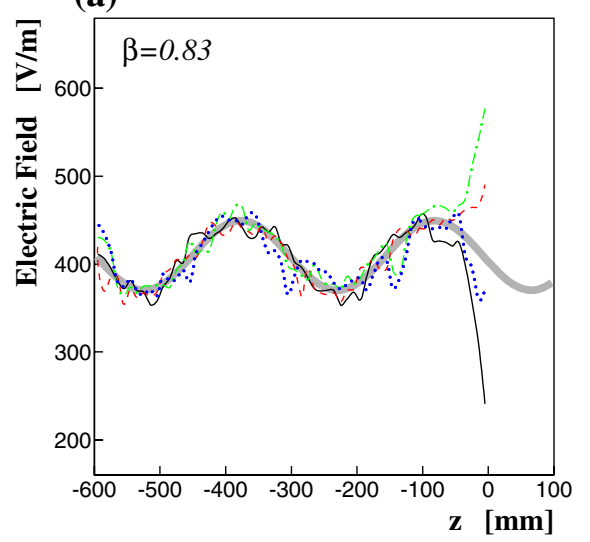

In the multipactoring simulation, a first electron is put on the surface of the inner or outer conductor with zero initial energy. Then, its relativistic motion is calculated by solving the second-order differential equation

$$
\frac{d^{2} \vec{x}}{d t^{2}}=\frac{e}{m_{e} \gamma}\left[\vec{E}+\frac{d \vec{x}}{d t} \times \vec{B}-\frac{1}{c^{2}}\left(\frac{d \vec{x}}{d t} \cdot \vec{E}\right) \frac{d \vec{x}}{d t}\right]
$$

using the Runge-Kutta-Nyström method [18], where the electromagnetic fields of the input TEM wave and the reflected TEM wave are taken into account:

$$
\begin{aligned}
E_{r}= & \frac{V_{\mathrm{a}}}{r}\left\{\sin \left[\omega\left(t-\frac{z-z_{\mathrm{ref}}}{c}\right)+\phi_{0}\right]\right. \\
& \left.+\Gamma \sin \left[\omega\left(t+\frac{z-z_{\mathrm{ref}}}{c}\right)+\phi_{0}\right]\right\}, \\
B_{\theta}= & \frac{V_{\mathrm{a}}}{c r}\left\{\sin \left[\omega\left(t-\frac{z-z_{\mathrm{ref}}}{c}\right)+\phi_{0}\right]\right. \\
& \left.-\Gamma \sin \left[\omega\left(t+\frac{z-z_{\mathrm{ref}}}{c}\right)+\phi_{0}\right]\right\}, \\
& E_{\theta}=E_{z}=B_{r}=B_{z}=0 .
\end{aligned}
$$

In the above Eqs. (1)-(3), $\vec{x}(t)$ indicates the position of the multipactoring electron, $e$ the electron charge, $m_{e}$ the electron mass, $c$ the speed of light in vacuum, $\phi_{0}$ the initial phase, $z_{\text {ref }}$ the $z$ position of the effective reflection plane, $\omega$ the angular frequency, $\gamma(t)=1 / \sqrt{1-|\vec{d}(t) / d t|^{2} / c^{2}}, r_{\text {in }}$ $\left(r_{\text {out }}\right)$ the radius of the inner (outer) conductor, $\vec{E}(\vec{x}, t)$ $[\vec{B}(\vec{x}, t)]$ the electric (magnetic) field vector, and $V_{\mathrm{a}}=$ $V_{\mathrm{p}} / \ln \left(r_{\text {out }} / r_{\text {in }}\right)$, where $V_{\mathrm{p}}$ indicates the peak voltage between the inner and outer conductors. In the case of the



FIG. 2. (Color) Standing-wave patterns of the electric field in the ARES coupler coaxial line at the resonance frequency for the coupling coefficient ( $\beta$ ) values of (a) 0.83 and (b) 2.4, where the $z$ region is extended to $z=-600 \mathrm{~mm}$, obtained using a threedimensional electromagnetic simulation. The finer solid, dashed, dash-dotted, and dotted lines indicate the patterns at $\theta=27^{\circ}, 117^{\circ}$, $207^{\circ}$, and $297^{\circ}$, respectively, and $r=27.6 \mathrm{~mm}$, where the coupling loop of the input coupler is set at $\theta=27^{\circ}$, simulating the ARES cavity with the multipactoring problem explained in Sec. I. There are some fine noisy structures due to the finite mesh sizes in the simulation. The gray bold lines are fit results using a sinusoidal function for the average of the four patterns in the region of $-600 \mathrm{~mm}<z<-50 \mathrm{~mm}$. The field strengths are normalized to the time-averaged input rf power of $1 \mathrm{~W}$. 
ARES coupler, the parameter values are $r_{\text {in }}=16.7 \mathrm{~mm}$, $r_{\text {out }}=38.5 \mathrm{~mm}$, and $\omega=2 \pi \times 508.9 \mathrm{MH} z$. The value of $z_{\text {ref }}$ was computed to be $+80 \mathrm{~mm}$ using a threedimensional electromagnetic simulation in order that the reflection coefficient could be treated as real in our model of multipactoring. Resultant shifts of the standing-wave patterns inward the cavity are seen in Fig. 2. The peak voltage is calculated using $V_{\mathrm{p}}=\sqrt{2 P_{\text {in }} Z}$, where $P_{\text {in }}$ indicates the time average of the input rf power, and the characteristic impedance of the coaxial line is $Z=$ $376.7 \Omega \times \ln \left(r_{\text {out }} / r_{\text {in }}\right) / 2 \pi=50 \Omega$. The time evolution is calculated with a step size of $2 \pi / \omega / 200(=9.8 \mathrm{ps})$. When an electron impacts the inner or outer conductor, the impact energy $\left(E_{\text {imp }}\right)$ is compared with the specified minimum $\left(E_{\min }=100 \mathrm{eV}\right)$ and maximum $\left(E_{\max }=1200 \mathrm{eV}\right)$ energies. If the impact energy is inside the range $E_{\min }<$ $E_{\text {imp }}<E_{\max }$, a secondary electron is launched, at the position where the primary one impacted on the conductor, with an initial energy of $5 \mathrm{eV}$ in the direction perpendicular to the conductor surface. Then, the same procedure is repeated, regarding the secondary electron as a primary one, until one of the following four conditions is satisfied: (i) the impact energy is outside the range, i.e. $E_{\text {imp }}<E_{\min }$ or $E_{\mathrm{imp}}>E_{\max }$; (ii) the electron is accelerated into the conductor in the first step just after it was put on, or launched from, the conductor; (iii) the multipactoring electron goes out of the coaxial-line region, i.e. $z<$ $-200 \mathrm{~mm}$ or $z>-50 \mathrm{~mm}$; (iv) the number of impacts exceeds a specified number, $N_{\max }=100$.

In this paper, multipactoring zones are represented using

$$
\begin{aligned}
\mathcal{M}\left(P_{\mathrm{in}}, \Gamma\right)= & \frac{1}{N_{\mathrm{p}}^{(z)} N_{\mathrm{p}}^{(\phi)}} \sum_{l=1}^{N_{\mathrm{p}}^{(z)}} \sum_{k=1}^{N_{\mathrm{p}}^{(\phi)}} \sum_{j=1}^{2} \frac{r_{j}}{r_{\mathrm{in}}+r_{\mathrm{out}}} \\
& \times f\left[N_{\mathrm{imp}}\left(P_{\mathrm{in}}, \Gamma ; j, k, l\right), 10\right],
\end{aligned}
$$

where $N_{\mathrm{p}}^{(z)}(=14)\left[N_{\mathrm{p}}^{(\phi)}(=180)\right]$ is the number of sampling points uniformly distributed along the $z$ axis (initial phase), $l$ is an index for the initial $z$ position $\left[z_{\text {ini }}=-150 \mathrm{~mm} \times\right.$ $\left.(l-1) /\left(N_{\mathrm{p}}^{(z)}-1\right)-50 \mathrm{~mm}\right], k$ an index for the initial phase $\left[\phi_{0}=2 \pi \times(k-1) / N_{\mathrm{p}}^{(\phi)}\right], j=1(j=2)$ means start from the inner (outer) conductor, $r_{1}=r_{\text {in }}, r_{2}=r_{\text {out }}$, $r_{j} /\left(r_{\text {in }}+r_{\text {out }}\right)$ is a weight concerning the surface areas of the inner and outer conductors, $N_{\text {imp }}$ indicates the number of impacts of the multipactoring electron, and the function $f$ is defined as

$$
f(x, a)= \begin{cases}x & (x \geq a) \\ 0 & (x<a)\end{cases}
$$

In the above definition of Eq. (5), the value of the secondary electron yield $(\delta)$ of the copper conductor is not considered; the range for $\delta>1$ is considered instead in the setting of $E_{\min }$ and $E_{\max }$ for $N_{\text {imp }}$ counting. There are other quantities used to represent multipactoring, e.g., see $[19,20]$.

\section{B. Definition}

A multipactoring zone map is defined as representing the quantity $\mathcal{M}$ as functions of the two observables, $P_{\text {in }}$ and $\Gamma$. Figure 3 shows an example for the coaxial line of the ARES input coupler. $\Gamma$ is calculated as the square root of the ratio of the reflected power $\left(P_{\text {ref }}\right)$ to $P_{\text {in }}: \Gamma=$ $\pm \sqrt{P_{\text {ref }} / P_{\text {in }}}$. The upper half of the map $(\Gamma>0)$ is a region where the coupler is being overcoupled to the electromagnetic field in the cavity, and the lower one $(\Gamma<0)$ for the coupler being undercoupled. An actual condition of a cavity with or without beam loading can be specified by a unique point in this map.

In Fig. 4(a), measurements of the input rf power and the reflection coefficient are superimposed for the ARES cavity with the multipactoring problem explained in Sec. I (we refer to this cavity as CavityM hereafter). The measurements were recorded when the HER accumulated a beam from zero current, so that the operating point started from the upper-left corner with $\Gamma \cong 0.5$ in the map, where the coupling-loop angle of the input coupler was set for the coupling coefficient of 3.0 before the KEKB operation. The rf power was fed into the cavity to obtain a specified cavity voltage of $0.27 \mathrm{MV}$. As the HER accumulated the beam current, the reflected power decreased due to beam loading. After traversing the optimum point with $\Gamma=0$, the operating point moved only inside a certain region near the huge multipactoring zone during the physics run in the

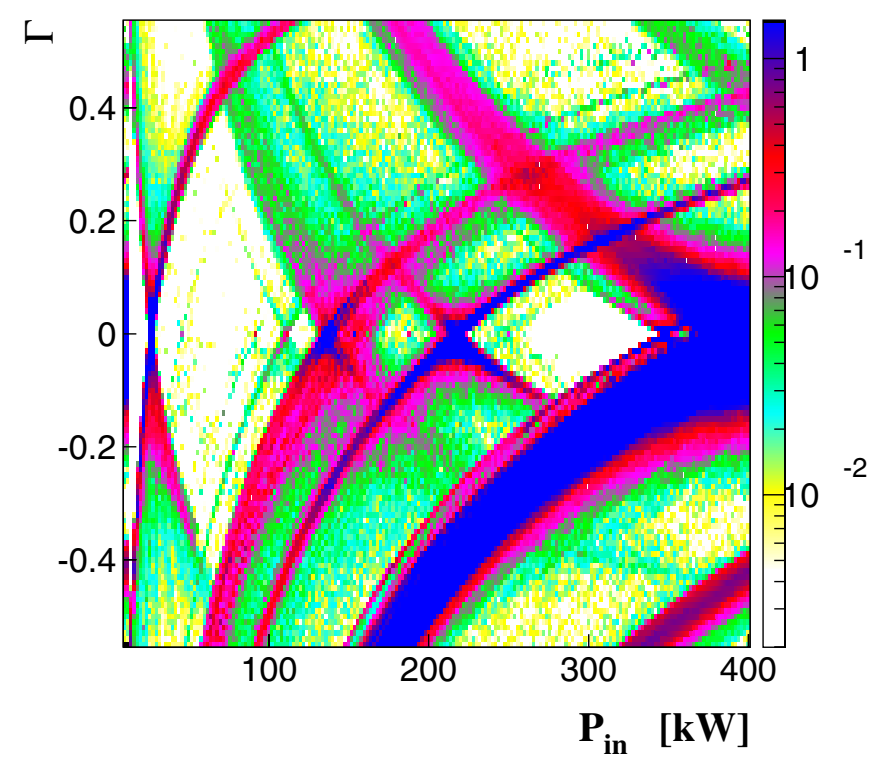

FIG. 3. (Color) Multipactoring zone map for the coaxial line of the ARES input coupler, showing the quantity $\mathcal{M}$ defined in Eq. (5). The horizontal axis indicates the time average of the input rf power, and the vertical one for the reflection coefficient. 
(a)

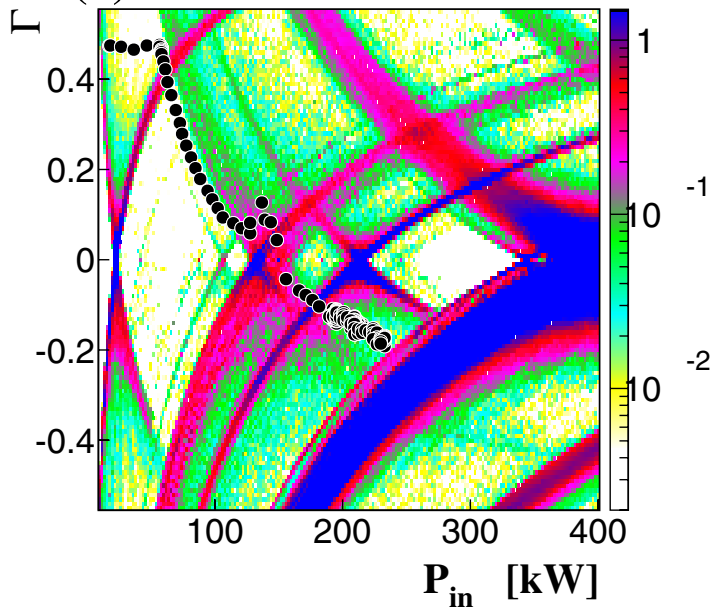

(c)



(e)

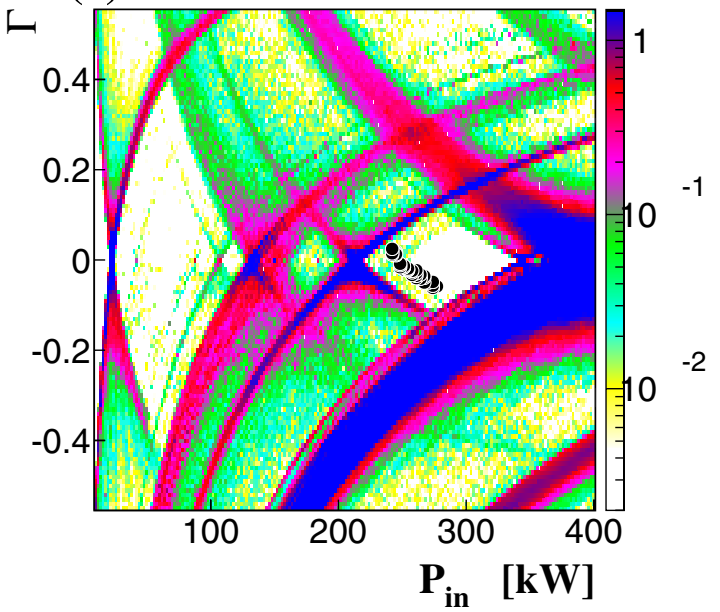

(b)

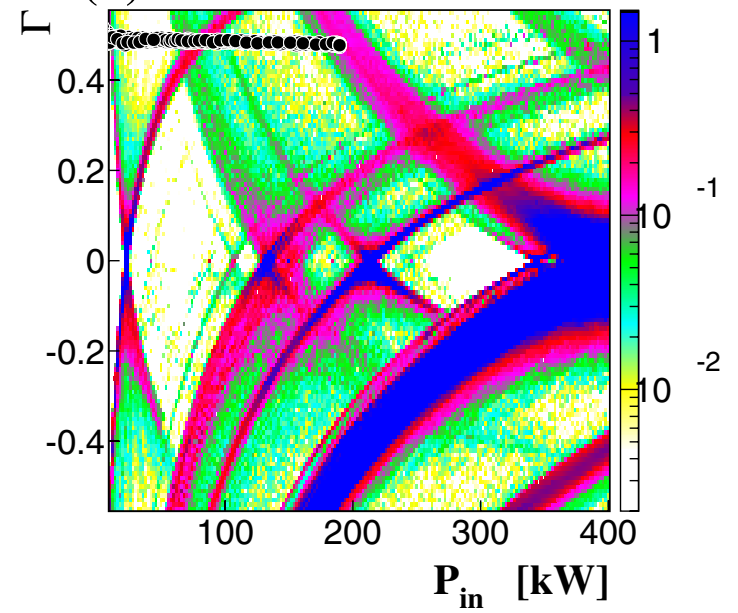

(d)

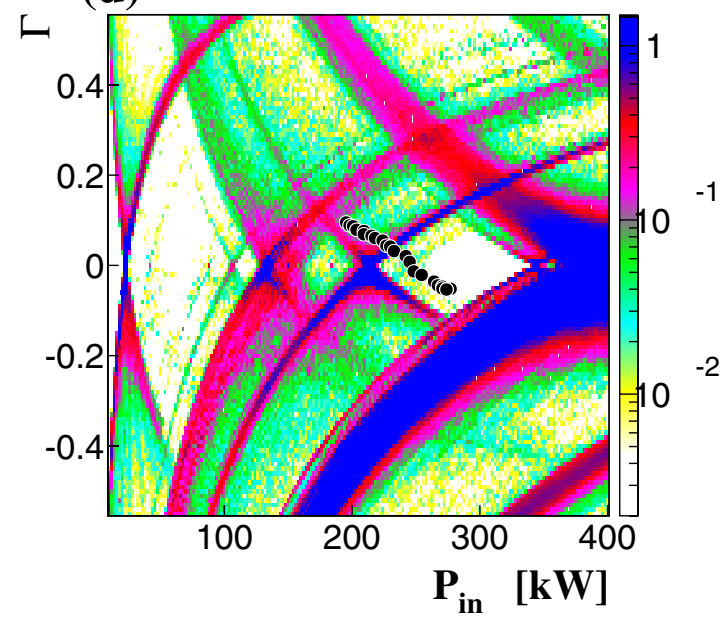

(f)

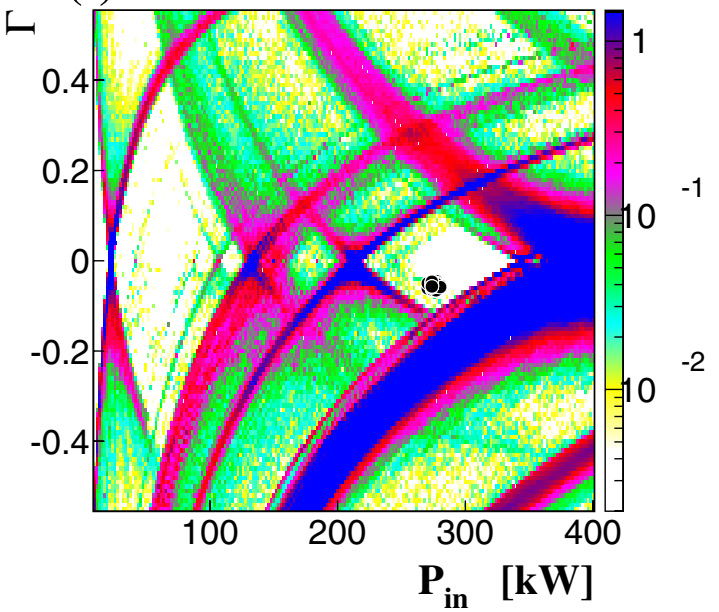

FIG. 4. (Color) Trajectories of the operating point of CavityM. The circles are measurements of the input rf power and the reflection coefficient in the periods of (a) the beam recovery, (b) the rf conditioning without a beam [for the comparison shown in Fig. 5(a)], (c) the beam decreasing without beam injection in a physics run [for the comparison shown in Fig. 5(b)], (d) the machine study to search for a new operating region [for the comparison shown in Fig. 5(c)], (e) the physics run in the CIM mode without the beam loading feedback, and (f) the physics run in the CIM mode with the beam loading feedback. The cavity voltage of CavityM was $0.27 \mathrm{MV}$ and $0.39 \mathrm{MV}$ for (a) and (c), respectively, and 0.34 MV for (d), (e), and (f). 
KEKB continuous injection mode (CIM). It should be noted that the measurement points are not on the $\Gamma=0$ line since there is a relatively large multipactoring zone at the optimum point.

\section{Comparisons with the vacuum data}

In order to check the validity of the multipactoring zone map, we compare the values of $\mathcal{M}$ with measurements of the vacuum pressure in CavityM, where vacuum pressures are measured using cold cathode gauges, and shown as equivalent nitrogen data. In Fig. 5(a), the vacuum data and the calculation result of $\mathcal{M}$ are shown as a function of the input rf power. This vacuum data was taken just after $\mathrm{rf}$ conditioning on a regular maintenance day. There are clear power-dependent pressure rises, which can be understood as being the operating point passing through, or approaching, the two multipactoring zone bands, as shown in Fig. 4(b).

In Fig. 5(b), the vacuum data and $\mathcal{M}$ during a physics run are shown as a function of the HER beam current. When this data was taken, the beam current decreased slowly without beam injection, from $1.2 \mathrm{~A}$ down to $0.5 \mathrm{~A}$ in three hours. There is a clear double-peak structure in the vacuum data, which can also be understood as being the operating point passing through the two multipactoring zone bands, as shown in Fig. 4(c).

(a)

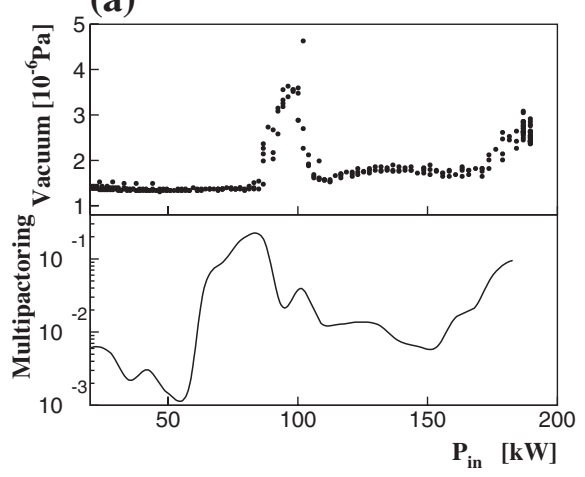

Quantitatively, all of the peak positions on $\mathcal{M}$ in Figs. 5(a) and 5(b) are shifted downward by about $10 \%$ compared with those in the vacuum data. However, the calibration accuracy of the rf power measurement, including the cable loss and the coupling strength of the directional coupler, is about $10 \%$; thus the agreements between the data and the simulation are good.

\section{OPERATIONAL STRATEGY AGAINST MULTIPACTORING}

We have applied the multipactoring zone map to the problem on CavityM mentioned in Sec. I. A prediction of the map is that the input coupler can be safely operated without multipactoring inside the diamond region around $P_{\text {in }}=280 \mathrm{~kW}$ and $\Gamma=0$. According to this map, we carried out a machine study to search for a new operating region, and found a candidate with a higher cavity voltage of $0.34 \mathrm{MV}$. Figure 5(c) shows how the vacuum pressure changes with the operating point stepping into the diamond region. As the multipactoring zone map predicts, the vacuum pressure becomes lower and the discharge in the coupler coaxial line disappears. CavityM now works with almost no multipactoring and no trip during physics runs in the CIM mode, where the operating point is almost always inside the multipactoring-free diamond region, as shown in Fig. 4(e).

(b)

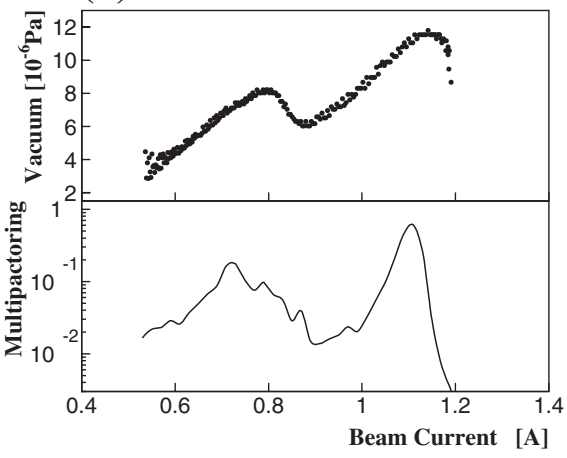

(c)

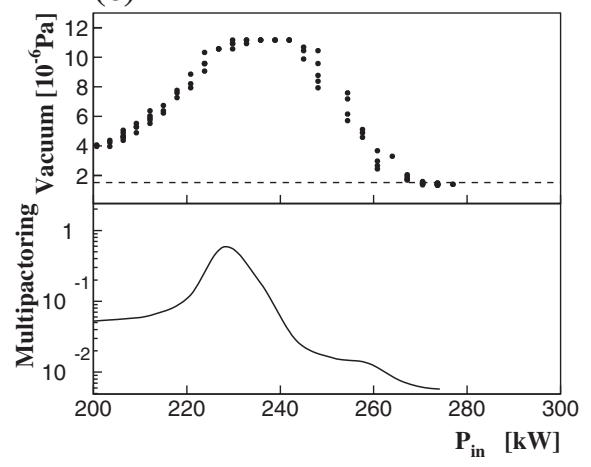

FIG. 5. Vacuum data of CavityM (upper) and calculation results of $\mathcal{M}$ (lower) as a function of the input rf power or the HER beam current. The dashed line in (c) indicates a typical vacuum pressure in the other cavities with no multipactoring problem. The trajectories of the operating point for (a), (b), and (c) are shown in Figs. 4(b)-4(d), respectively. 

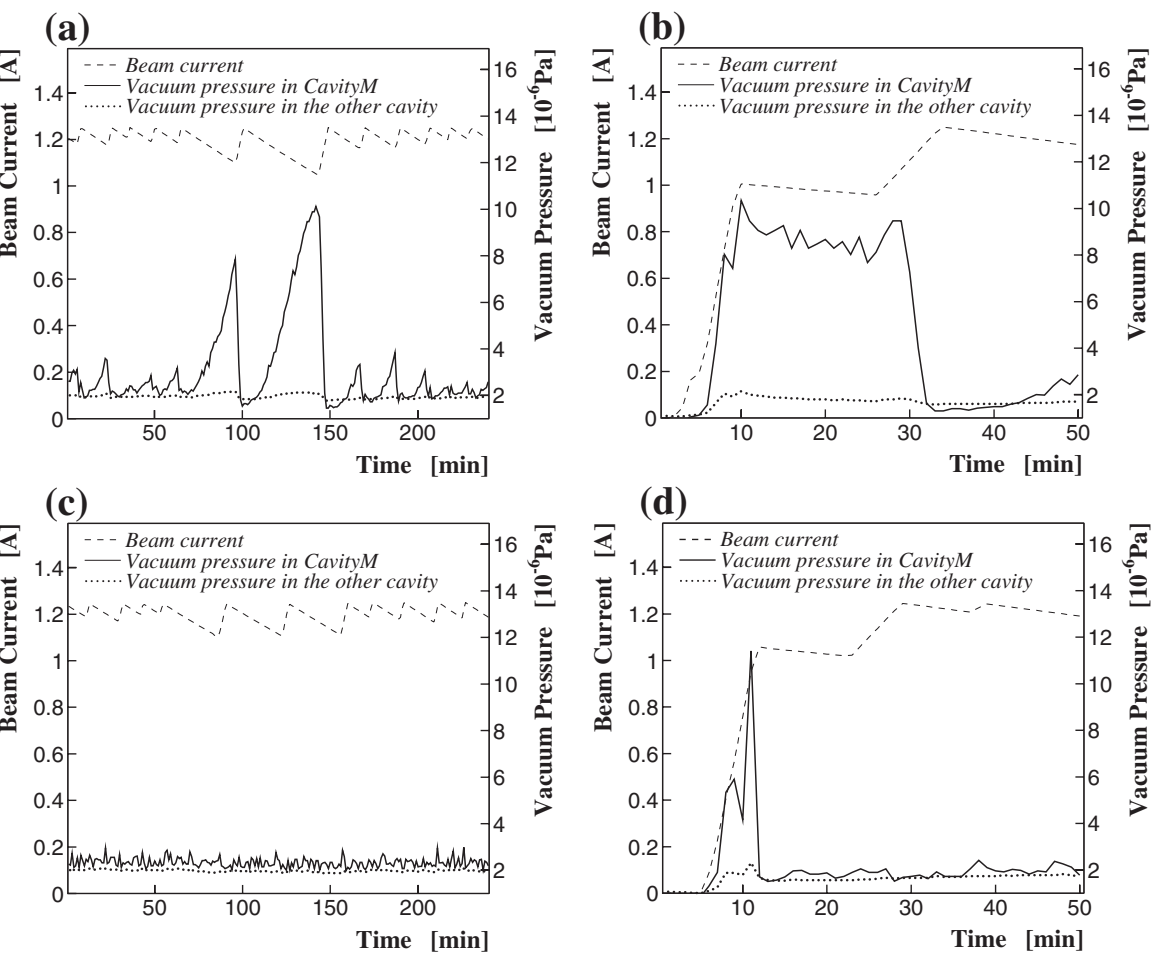

FIG. 6. Vacuum-pressure histories of CavityM (solid lines) and of the other cavity which has no multipactoring problem (dotted lines), together with histories of the HER beam current (dashed lines), for the periods of (a) the physics run in the CIM mode without the beam loading feedback [the trajectory of the operating point is shown in Fig. 4(e)], (b) the beam recovery without the beam loading feedback [the trajectory of the operating point for the early 30-five minutes is shown in Fig. 8(a)], (c) the physics run in the CIM mode with the beam loading feedback [the trajectory of the operating point is shown in Fig. 4(f)], and (d) the beam recovery with the beam loading feedback [the trajectory of the operating point for the early 15 minutes is shown in Fig. 8(b)].

However, there is a remaining problem: the beam current sometimes decreases for some reasons, which results in a situation where the operating point approaches the multipactoring zone band around $P_{\text {in }}=240 \mathrm{~kW}$. Figure 6(a) shows an example of this kind of problem. Another example is shown in Fig. 6(b), which is recovery from a total-beam loss. The vacuum pressure became significantly higher when the operating point was passing through the multipactoring zone.

Considering the fact that cavities out of condition are in a minority (one or two of the 12 ARES cavities together with the eight SCCs for the HER), we can keep the input rf power in the multipactoring-free diamond region by changing the beam loading of the troubling cavity, equivalently, by changing the rf phase of the cavity relative to the beam. In this case, an additional positive or negative beam loading is distributed among the other ten ARES cavities and eight SCCs, where one klystron drives the two ARES cavities of CavityM and the other with the rf power divided equally between the two by a magic $\mathrm{T}$ in the current configuration of the KEKB-rf system. However, the amount of the additional beam loading should be small enough to be negligible. The above-mentioned procedure is to be done automatically using a computer program as a beam loading feedback.
The target input-power region is determined by scanning the vacuum pressure inside the diamond region. Figure 7 shows the vacuum data as a function of the input rf power.

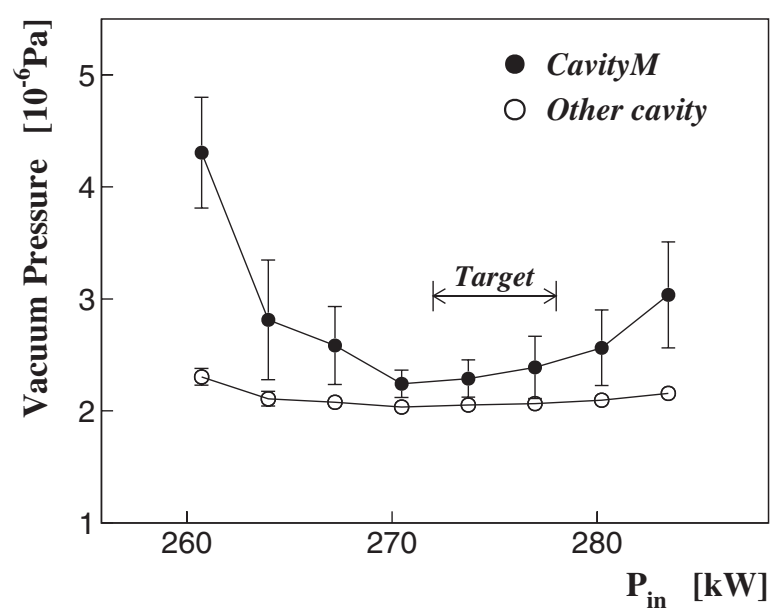

FIG. 7. Vacuum pressures in CavityM and in the other cavity which has no multipactoring problem. Each point is an average of about ten measurements, and the vertical bars indicate a statistical error. The horizontal arrow denotes the target power region for the beam loading feedback. 
(a)

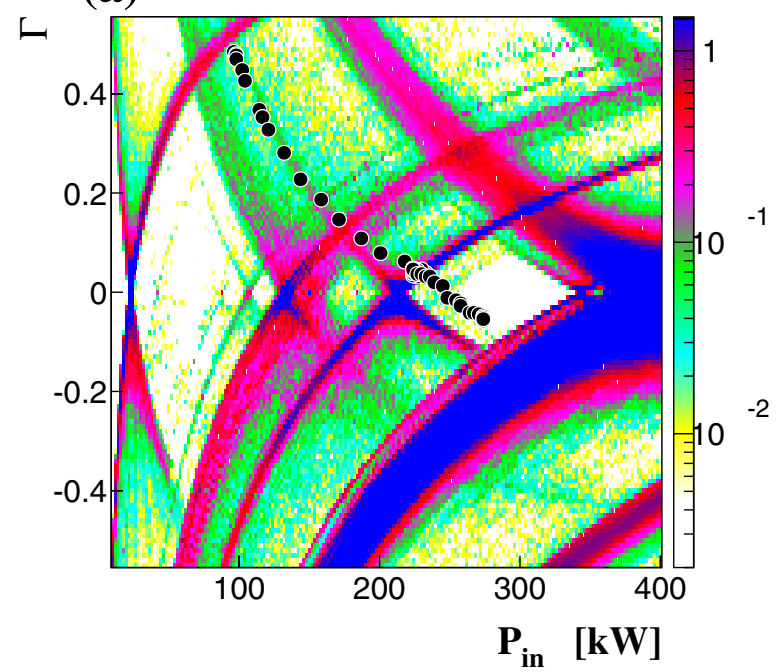

(b)

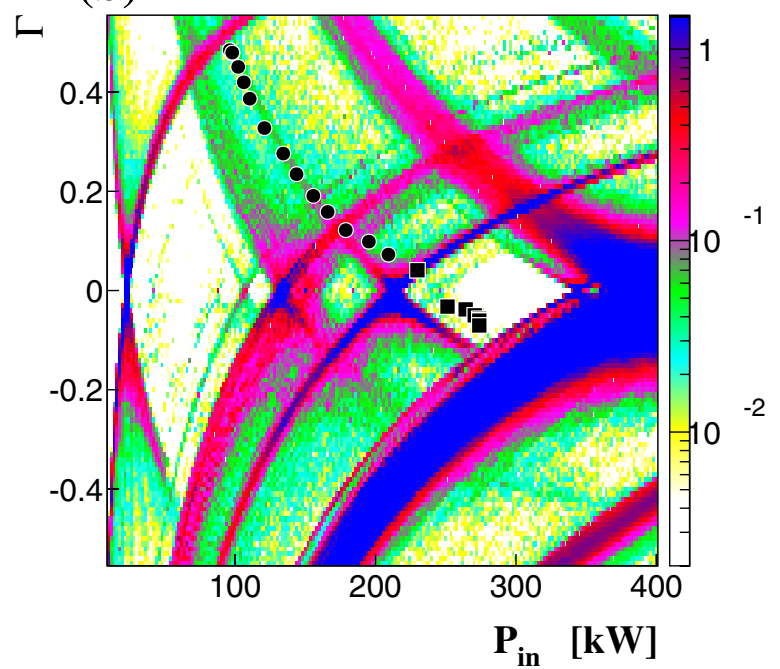

FIG. 8. (Color) Trajectories of the operating point of CavityM in the periods of (a) the beam recovery without the beam loading feedback for the early 30-five minutes in Fig. 6(b), and (b) the beam recovery with the beam loading feedback for the early 15 minutes in Fig. 6(d). The circles are data without the feedback, and the squares by using the feedback with the beam current above the threshold ( 0.9 A) for activating the feedback. The measurements of the circles and squares were recorded every 30 seconds.

We set the region of $272 \mathrm{~kW}<P_{\text {in }}<278 \mathrm{~kW}$ as a target for the feedback.

The feedback is executed every five seconds according to the following logic. The beam loading is regulated by changing the cavity phase in order the input rf power to change by (i) $+1.5 \mathrm{~kW}$ for $P_{\text {in }}<266 \mathrm{~kW}$, (ii) $+1.0 \mathrm{~kW}$ for $266 \mathrm{~kW}<P_{\text {in }}<270 \mathrm{~kW}$, (iii) $+0.5 \mathrm{~kW}$ for $270 \mathrm{~kW}<P_{\text {in }}<272 \mathrm{~kW}$, (iv) $-0.8 \mathrm{~kW}$ for $278 \mathrm{~kW}<$ $P_{\text {in }}<282 \mathrm{~kW}, \quad(\mathrm{v})-1.5 \mathrm{~kW}$ for $282 \mathrm{~kW}<P_{\text {in }}<$ $285 \mathrm{~kW}$, and (vi) $-2.5 \mathrm{~kW}$ for $P_{\text {in }}>285 \mathrm{~kW}$. This feedback is activated only when there is an input rf power into
CavityM and the beam current is higher than $0.65 \mathrm{~A}$ (about a half of the maximum beam current). For safety, the beam loading should be set to be light enough just after a totalbeam loss.

With this feedback, the vacuum pressure during physics runs has become independent of the beam current, and comparable to those in the other cavities, as shown in Fig. 6(c), where the area of the operating point is localized inside the diamond, as shown in Fig. 4(f). Furthermore, the time spent to pass through the multipactoring zone in beam recovery has been shortened due to a quick passage, as shown in Fig. 8. As a result, the time with higher vacuum pressures has also been shortened, as shown in Fig. 6(d).

\section{CONCLUSIONS}

Since the reflection coefficient varies widely with the beam loading in the case of high beam current storage rings, multipactoring zones should be represented in the two-dimensional space of the input rf power and the reflection coefficient. We have performed a simulation study for the KEKB/ARES based on this map, and solved the multipactoring problem by finding a new operating region without multipactoring. This fact demonstrates the usefulness of the multipactoring zone map.

Furthermore, based on this map, we have developed a method to operate an rf cavity suffering from multipactoring; the operating point is automatically controlled, by a computer program which changes the cavity phase, to be in a target input-power region where the vacuum pressure is around the minimum and there is no multipactoring. This beam loading feedback program has been successfully running during the KEKB operations. It should be noted that this is a temporary measure, for the KEKB performance not to be degraded, until a final measure will be taken; e.g., development of a multipactoring-free coupler, where the multipactoring zone map should be useful for designing.

\section{ACKNOWLEDGMENTS}

The authors are grateful to Hiromi Hisamatsu for giving us calibration data of the cold cathode gauge. The authors are also grateful to the other members of the KEKB group for their fruitful comments and various support.

[1] T. Kageyama et al., in Proceedings of the 1st Asian Particle Accelerator Conference (APAC 98), Tsukuba, Japan, 1998 (KEK Preprint No. 98-45).

[2] Y. Yamazaki and T. Kageyama, Part. Accel. 44, 107 (1994).

[3] K. Akai et al., Nucl. Instrum. Methods Phys. Res., Sect. A 499, 45 (2003).

[4] S. Kurokawa, Nucl. Instrum. Methods Phys. Res., Sect. A 499, 1 (2003). 
[5] M. Kikuchi et al., Nucl. Instrum. Methods Phys. Res., Sect. A 499, 8 (2003).

[6] K. Egawa et al., Nucl. Instrum. Methods Phys. Res., Sect. A 499, 24 (2003).

[7] K. Kanazawa et al., Nucl. Instrum. Methods Phys. Res., Sect. A 499, 66 (2003).

[8] K. Kanazawa et al., Nucl. Instrum. Methods Phys. Res., Sect. A 499, 75 (2003).

[9] M. Arinaga et al., Nucl. Instrum. Methods Phys. Res., Sect. A 499, 100 (2003).

[10] N. Akasaka et al., Nucl. Instrum. Methods Phys. Res., Sect. A 499, 138 (2003).

[11] I. Abe et al., Nucl. Instrum. Methods Phys. Res., Sect. A 499, 167 (2003).

[12] K. Akai et al., Nucl. Instrum. Methods Phys. Res., Sect. A. 499, 191 (2003).

[13] F. Naito et al., in Proceedings of the 1st Asian Particle Accelerator Conference (APAC 98), Tsukuba, Japan, 1998
(KEK Preprint No. 98-44).

[14] H. Sakai et al., (2005), in the 2005 Particle Accelerator Conference (PAC 05), Knoxville, Tennessee, USA, 2005 (Report No. TPPT012).

[15] J. Tuckmantel et al., in The 16th Particle Accelerator Conference-PAC 95, Dallas, TX, USA, 1995; and International Conference on High Energy Accelerators (IUPAP).

[16] P. Yla-Oijala, DESY-TESLA-97-21, 1997.

[17] R. Geng, H. Padamsee, and V. Shemelin (2001), in IEEE Particle Accelerator Conference (PAC2001), Chicago, Illinois, 2001.

[18] L. Collatz, The Numerical Treatment of Differential Equations (Springer-Verlag, Berlin, 1960).

[19] Y. Kijima, in The 10th workshop on RF superconductivity, 2001 (Report No. PT028).

[20] C. Schulz, CERN-THESIS-2004-008, 2004. 